\title{
New Series Expansions for the 3-D Green's Function of Multilayered Media With 1-D Periodicity Based on Perfectly Matched Layers
}

\author{
Hendrik Rogier, Senior Member, IEEE
}

\begin{abstract}
A new formalism based on perfectly matched layers (PMLs) is presented to derive new series expansions for the Green's function of an infinite set of point sources with a 1-D periodicity embedded in a layered medium. Several PML-based series expansions, both in the spatial and spectral domains, combined with suitable convergence acceleration techniques such as the Shanks transform and Ewald transform, are proposed and their efficiency is evaluated. For each pair of excitation and observation locations, an optimal series expansion in terms of accuracy and CPU time is proposed, resulting in a significant speed-up compared to existing approaches.
\end{abstract}

Index Terms-Green's functions for multilayered media, integral-equation techniques, perfectly matched layers (PMLs), periodic structures.

\section{INTRODUCTION}

$\mathbf{M}$ ANY practical waveguiding, scattering, and radiating devices, such as gratings [1]-[3], arrays [4], metamaterials and electromagnetic (EM)-bandgap structures [5]-[7], nonradiating dielectric waveguides [8], ..., can be efficiently modeled as 2-D configurations [1]-[3], [5], [6], or 3-D configurations [4], [7], [8] with 1-D periodicity [1]-[3], 2-D periodicity [4], [6], or 3-D periodicity [7]. Moreover, several configurations relevant in the analysis of EM field propagation, such as EM shieldings [9] and reinforced concrete walls [10], [11], are well approximated by a periodic structure with infinite extent. By applying the Floquet-Bloch theorem, the analysis of a periodic structure with infinite extent is restricted to a representative unit cell. For the description of the fields by means of an integral-equation technique, the periodic Green's function is required to account for the periodic character of the configuration, as discussed in Section II. The Green's function approach is especially efficient for a configuration of scatterers embedded in a planar multilayered dielectric background medium since the effect of the background medium can be fully incorporated into the Green's function. However, in order to determine the EM fields of a single point source located in the layered medium, a time-consuming Sommerfeld integration is required for the inverse Hankel transform that transforms the analytical solution from the spectral domain to spatial domain.

\footnotetext{
Manuscript received February 7, 2007; revised April 23, 2007. This work was supported by the Fund for Scientific Research-Flanders (FWO-V).

The author is with the Information Technology Department, Ghent University, B-9000 Ghent, Belgium (e-mail: hendrik.rogier@intec.UGent.be).

Digital Object Identifier 10.1109/TMTT.2007.902580
}

The periodic Green's function is written as a spatial-domain series or as a spectral-domain series of the field solution for the single point source. For a 2-D periodic grid of point sources, the spectral-domain series can be expressed in an analytical way and Sommerfeld integrations are avoided. In this paper, however, we consider a 1-D periodic grid of point sources. In that case, even for the spectral-domain series, an inverse Fourier transform is required, as demonstrated in Section III-A. An additional complication results from the fact that both spectral- and spatial-domain series tend to be slowly converging for certain positions of the excitation and the observation point. Therefore, much attention has been devoted to derive series expansions that converge more rapidly, mainly by combining both the spatial- and spectral-domain series. Convergence acceleration techniques were proposed in literature for the 1-D periodic 2-D Green's function [12]-[16] and for the 2-D periodic 3-D Green's function. In [17], we presented a new efficient approach to calculate the 1-D periodic 2-D Green's function based on the use of perfectly matched layers (PMLs). Up to now, little has been published about the 1-D periodic 3-D Green's function. In [18] and [19], the 1-D periodic 3-D Green's function for a periodic set of point sources located in free space is accelerated by means of the Ewald transform. However, an extension of this approach in order to include a stratified dielectric background medium is not straightforward. In [20], the 1-D periodic 3-D Green's functions for a microstrip substrate are derived in the spectral domain first, and the corresponding spatial-domain quantities are obtained through an efficient sum of inverse Fourier transforms.

In this paper, a new formalism based on PMLs is proposed to derive a fast converging series expansion for the 1-D periodic 3-D Green's function of layered media. As in [21]-[25], PMLs [26]-[29] are used to transform the open layered medium into a closed waveguide configuration. An efficient expansion for the 3-D Green's function of a point source in the stratified background medium in terms of a set of discrete modes of the closed waveguide containing the PML is then possible, while the PMLs mimic the open character. For a theoretical background about issues of completeness and convergence, the reader is referred to [30] and [31]. Based on the PML-based modal expansion, analytical series expansions are then derived in Section III-B. As both the spectral- and spatial-domain series suffer from slow convergence, special attention is devoted in Section III-C to accelerate convergence of the PML-based series. This acceleration is based on the use of the Shanks transform and the Ewald transform. Moreover, as in some cases, the accuracy and efficiency of the PML-based series is not ensured in the singularity 
region (when source and observation point are close to each other), a hybrid series is derived in Section III-C that combines the classic nonperiodic Green's function, capturing the correct singularity, with a PML-based series for the remaining periodic part. In Section IV, we validate the new series and evaluate their accuracy and efficiency in terms of CPU time. For each combination of excitation and observation locations, an optimal series expansion in terms of accuracy and CPU time is proposed.

\section{INTEGRAL EQUATION FOR A 1-D PERIODIC CONFIGURATION OF CONDUCTORS IN A LAYERED MEDIUM}

Consider a planar stratified medium of $N$ dielectric layers with finite thickness along the $z$-direction, but of infinite extent in the $x$ - and $y$-directions. The stack of dielectric layers can be backed by a perfect electrically conducting ground plane. A 1-D periodic 3-D configuration of planar conductors, located in different layers parallel to the $x y$-plane and infinitely thin in the $z$-direction, can be described by means of the mixed potential integral equation

$$
\begin{aligned}
-\mathbf{e}_{\mathrm{tan}}^{\mathrm{exc}}(\mathbf{r})=-j & \omega \mu \int_{S} G_{A}^{\mathrm{per}, 1-\mathrm{D}}\left(\mathbf{r} ; \mathbf{r}^{\prime}\right) \mathbf{j}\left(\mathbf{r}^{\prime}\right) d S^{\prime} \\
& +\frac{1}{j \omega \epsilon} \int_{S} \nabla_{t} G_{V}^{\mathrm{per}, 1-\mathrm{D}}\left(\mathbf{r} ; \mathbf{r}^{\prime}\right) \nabla_{t}^{\prime} \cdot \mathbf{j}\left(\mathbf{r}^{\prime}\right) d S^{\prime}
\end{aligned}
$$

provided that we are able to determine the kernel functions $G_{A}^{\text {per, } 1-\mathrm{D}}\left(\mathbf{r} ; \mathbf{r}^{\prime}\right)$ and $G_{V}^{\text {per }, 1-\mathrm{D}}\left(\mathbf{r} ; \mathbf{r}^{\prime}\right)$ that account for both the 1-D periodicity and the presence of the multilayered background medium. The excitation $\mathbf{e}_{\tan }^{\text {exc }}(\mathbf{r})$ represents the component tangential to the $x y$-plane of the electric field response of the background medium to an imposed electric field, which can, e.g., be an incident plane wave of the form $\mathbf{e}^{i}(\mathbf{r})=e^{-j\left(k_{x} x+k_{y} y+k_{z} z\right)} \mathbf{u}$. The solution of (1) yields the surface current distribution $\mathbf{j}\left(\mathbf{r}^{\prime}\right)=j_{x}\left(x^{\prime}, y^{\prime}, z^{\prime}\right) \mathbf{u}_{x}+j_{y}\left(x^{\prime}, y^{\prime}, z^{\prime}\right) \mathbf{u}_{y}$ on all planar conductors. By considering only planar conductors, the Green's function dyadic $\overline{\bar{G}}_{A}^{\text {per,1-D }}\left(\mathbf{r} ; \mathbf{r}^{\prime}\right)$ was reduced to a single component $G_{A}^{\text {per, } 1-\mathrm{D}}\left(\mathbf{r} ; \mathbf{r}^{\prime}\right)$ in $(1)$.

\section{1-D PERIODIC 3-D GREEN'S FUNCTIONS $G_{A}^{\text {per,1-D }}$ AND $G_{V}^{\text {per,1-D }}$}

\section{A. Classic Green's Function Series}

Consider a planar multilayered dielectric background medium in which we place a 1-D grid of point sources. We assume that the problem is periodic in the $x$-direction with the period given by $b$; two adjacent point source excitations differ by, at most, a phase factor $e^{-j k_{x} b}$. The conventional approach of finding the 3-D Green's function for this 1-D periodic configuration proceeds by first determining the spatial-domain Green's functions $G_{A}\left(x, y, z ; x^{\prime}, y^{\prime}, z^{\prime}\right)$ and $G_{V}\left(x, y, z ; x^{\prime}, y^{\prime}, z^{\prime}\right)$ for a single point source of elementary current and charge, respectively. In order to evaluate these Green's functions in a multilayered medium, a time-consuming Sommerfeld integration is required to convert the spectral-domain Green's functions $\tilde{G}_{A, V}\left(k_{x}, k_{y}, z ; z^{\prime}\right)$ to the spatial domain. Moreover, the resulting series

$$
\begin{aligned}
G_{A, V}^{\mathrm{per}, 1-\mathrm{D}} & \left(x, y, z ; x^{\prime}, y^{\prime}, z^{\prime}\right) \\
& =\sum_{m=-\infty}^{m=+\infty} e^{-j k_{x} m b} G_{A, V}\left(x, y, z ; x^{\prime}+m b, y^{\prime}, z^{\prime}\right)
\end{aligned}
$$

is known to be slowly convergent so many spatial Green's function evaluations are required in order to obtain an accurate result.

In free space, (2) reduces to

$$
\begin{aligned}
G_{A, V}^{\mathrm{per}, 1-\mathrm{D}} & \left(x, y, z ; x^{\prime}, y^{\prime}, z^{\prime}\right) \\
& =\sum_{m=-\infty}^{m=+\infty} \frac{e^{-j k_{x} m b} e^{-j k_{o}} \sqrt{\left(x-x^{\prime}-m b\right)^{2}+\left(y-y^{\prime}\right)^{2}+\left(z-z^{\prime}\right)^{2}}}{4 \pi \sqrt{\left(x-x^{\prime}-m b\right)^{2}+\left(y-y^{\prime}\right)^{2}+\left(z-z^{\prime}\right)^{2}}}
\end{aligned}
$$

with $k_{0}^{2}=\omega^{2} \epsilon_{0} \mu_{0}$, and the Poisson transform can be applied to obtain the spectral-domain analog on

$$
\begin{aligned}
& G_{A, V}^{\mathrm{per}, 1-\mathrm{D}}\left(x, y, z ; x^{\prime}, y^{\prime}, z^{\prime}\right) \\
& =\frac{1}{4 j b} \sum_{m=-\infty}^{m=+\infty} e^{-j k_{x, m}\left(x-x^{\prime}\right)} \\
& \quad \times H_{0}^{(2)}\left(\Gamma_{x, m} \sqrt{\left(y-y^{\prime}\right)^{2}+\left(z-z^{\prime}\right)^{2}}\right)
\end{aligned}
$$

with $k_{x, m}=k_{x}+(2 \pi m / b)$ and $\Gamma_{x, m}=\sqrt{k_{0}^{2}-k_{x, m}^{2}}$. In this case, both spectral- and spatial-domain series can be combined efficiently using the Ewald transform in order to obtain two fast converging series [18], [19].

In the general case of a multilayered background medium, however, no analytical expressions are available for the terms in the spectral-domain series [20]

$$
\begin{aligned}
& G_{A, V}^{\mathrm{per}, 1-\mathrm{D}}\left(x, y, z ; x^{\prime}, y^{\prime}, z^{\prime}\right) \\
& =\frac{1}{2 \pi b} \sum_{m=-\infty}^{m=+\infty} e^{-j k_{x, m}\left(x-x^{\prime}\right)} \\
& \times \int_{-\infty}^{+\infty} \tilde{G}_{A, V}\left(k_{x, m}, k_{y}, z ; z^{\prime}\right) e^{-j k_{y}\left(y-y^{\prime}\right)} d k_{y} .
\end{aligned}
$$

Now, a time-consuming inverse Fourier transform must be evaluated for each term in the spectral series. In [20], a fundamental speedup is obtained by extracting the singular behavior and by transforming that part into two fast converging series by means of the Kummer and Poisson transforms.

\section{B. Series Based on PMLs}

Assume that the background medium (Fig. 1) is translation invariant in the $x$ - and $y$-direction (a planar stratified medium) and that all material variations in the $z$-direction are located in a region that is bounded in that direction. The largest distance over which material variations extend in the $z$-direction is denoted by $t$ (in Fig. 2, where a single-layered microstrip substrate is shown, this coresponds to the thickness $d$ of the substrate). For a faster evaluation of the 1-D periodic 3-D Green's functions, we 


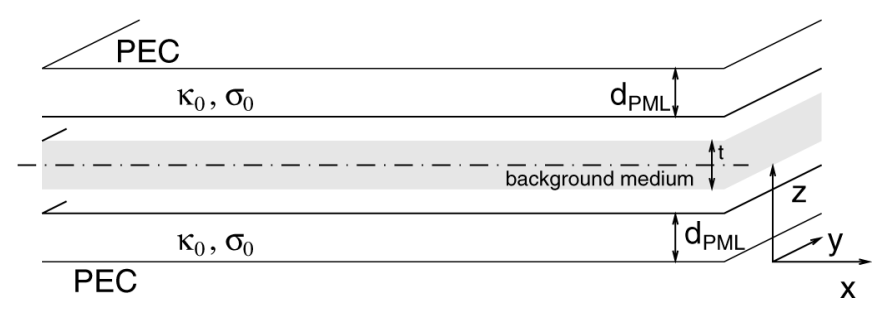

Fig. 1. Pertinent to the calculation of the Green's function.

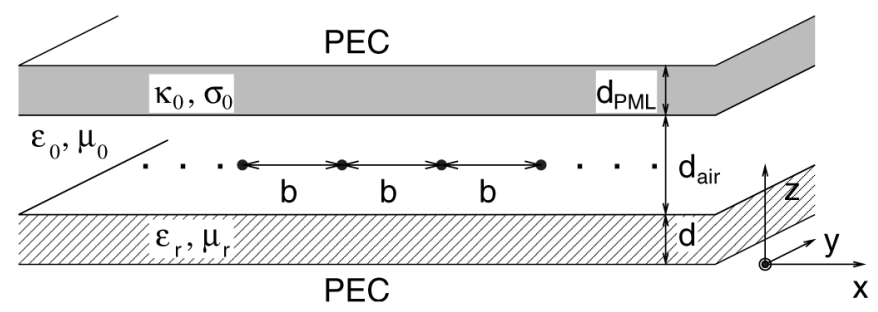

Fig. 2. 1-D periodic configuration of point sources on a microstrip substrate terminated by a PML.

then construct a parallel-plate waveguide by terminating the free space with two perfect electrically conducting plates backed by an isotropic PML with thickness $d_{\mathrm{PML}}$ and with material parameters $\kappa_{0}$ and $\sigma_{0}$ [32], as shown in Fig. 1. As the constitutive parameters are chosen the same as for the air layer, the isotropic PML can be combined with the air region to form a new air region with a complex thickness. A similar approach also applies to a planar stratified medium above a ground plane, as for the microstrip substrate in Fig. 2.

As in [21] and [22], the 3-D Green's function can be expanded into a series of discrete eigenmodes of the resulting parallelplate waveguide in the following way:

$$
\begin{aligned}
& G_{A, V}\left(x, y, z ; x^{\prime}, y^{\prime}, z^{\prime}\right) \\
&= \frac{1}{4 j} \sum_{n=1}^{+\infty} A_{n}\left(\beta_{n}, z \mid z^{\prime}\right) \\
& \quad \times H_{0}^{(2)}\left(\beta_{n} \sqrt{\left(x-x^{\prime}\right)^{2}+\left(y-y^{\prime}\right)^{2}}\right)
\end{aligned}
$$

with $\beta_{n}$ being the eigenvalues and with $A_{n}\left(\beta_{n}, z \mid z^{\prime}\right)$ being the excitation coefficients of the eigenmodes. Note that, in a free-space environment terminated by two PMLs forming a waveguide of total thickness $\tilde{d}=$ $d_{\text {air }}+2 d_{\mathrm{PML}}\left(\kappa_{0}-j\left(\sigma_{0} / \omega \epsilon_{0}\right)\right)$, the eigenvalues are given by $\beta_{n}=\sqrt{k_{0}^{2}-(n \pi / \tilde{d})^{2}}$ and the excitation coefficients are given by $A_{n}\left(\beta_{n}, z \mid z^{\prime}\right)=(2 / \tilde{d}) \sin ((n \pi / \tilde{d}) z) \sin \left((n \pi / \tilde{d}) z^{\prime}\right)$, where the origin for $z$ and $z^{\prime}$ is chosen at the bottom PEC plate (Fig. 1). For a microstrip substrate of thickness $d$ (Fig. 2), we distinguish between TE and TM eigenmodes [33]. The eigenvalues of the TE and TM modes are the solution of

$$
Y_{r, n} \cot \left(\gamma_{r, n} d\right)=-Y_{0, n} \cot \left(\gamma_{0, n} \tilde{d}\right)
$$

with $\gamma_{0, n}^{2}=k_{0}^{2}-\beta_{n}^{2}, \gamma_{r, n}^{2}=k_{0}^{2} \epsilon_{r} \mu_{r}-\beta_{n}^{2}$, and $k_{0}^{2}=\omega^{2} \epsilon_{0} \mu_{0}$, with $Y_{r, n, \mathrm{TE}}=\gamma_{r, n} / j \omega \mu_{0} \mu_{r}$ and $Y_{0, n, \mathrm{TE}}=\gamma_{0, n} / j \omega \mu_{0}$ for the TE case, and $Y_{r, n, \mathrm{TM}}=j \omega \epsilon_{0} \epsilon_{r} / \gamma_{r, n}$ and $Y_{0, n, \mathrm{TM}}=$ $j \omega \epsilon_{0} / \gamma_{0, n}$ for the TM case. For both TE and TM modes, the propagation constants of the leaky modes (which concentrate in the microstrip substrate) and of the Berenger modes (which concentrate in the PML) can be determined very rapidly based on analytical approximations derived in [34]. Given the initial estimates, the exact locations of the propagation constants of the TE and TM modes are found by performing a few iterations with Newton's method, as described in [35]. For general multilayered substrates, this technique is applicable for the Berenger modes only. The leaky modes are then determined based on the complex root finders proposed in [36].

In (6), the Green's function $G_{A}\left(x, y, z ; x^{\prime}, y^{\prime}, z^{\prime}\right)$ can be expanded into the TE modes of the closed PML-waveguide, and the excitation coefficients of the TE eigenmodes in or on top of the substrate $(0<z \leq d)$ are given by (8), shown at the bottom of this page. ${ }^{1}$ No TM modes are needed.

As for the Green's function $G_{V}\left(x, y, z ; x^{\prime}, y^{\prime}, z^{\prime}\right)$, (6) is composed of both TE and TM modes. The excitation coefficients of the TE eigenmodes in or on top of the substrate $(0<z \leq d)$ are given in (9), shown at the bottom of this page, ${ }^{1}$ whereas the excitation coefficients of the TM eigenmodes in or on top of the substrate $(0<z \leq d)$ are given by (10), shown at the bottom of the following page. ${ }^{1}$

\footnotetext{
${ }^{1}$ The index $n$ is omitted for simplicity of notation.
}

$$
A_{n}\left(\beta_{n}, z \mid z^{\prime}\right)=2 \frac{\sin \left(\gamma_{r} z\right) \sin \left(\gamma_{r} z^{\prime}\right)}{\frac{d}{\mu_{r}}+\tilde{d} \frac{Y_{0}^{2}+Y_{r}^{2}}{2 Y_{0}^{2}}-\frac{\gamma_{0}^{2}-\gamma_{r}^{2}}{\gamma_{0} Y_{0} \mu_{r}} \frac{\sin \left(2 \gamma_{r} d\right)}{2 \gamma_{r}}-\frac{Y_{0}^{2}-Y_{r}^{2}}{Y_{0}^{2}} \frac{\tilde{d}}{2} \cos \left(2 \gamma_{r} d\right)}
$$

$$
A_{n}\left(\beta_{n}, z \mid z^{\prime}\right)=\frac{2 k_{0}^{2}}{\beta^{2}} \frac{\sin \left(\gamma_{r} z\right) \sin \left(\gamma_{r} z^{\prime}\right)}{\frac{d}{\mu_{r}}+\tilde{d} \frac{Y_{0}^{2}+Y_{r}^{2}}{2 Y_{0}^{2}}-\frac{\gamma_{0}^{2}-\gamma_{r}^{2}}{\gamma_{0} Y_{0} \mu_{r}} \frac{\sin \left(2 \gamma_{r} d\right)}{2 \gamma_{r}}-\frac{Y_{0}^{2}-Y_{r}^{2}}{Y_{0}^{2}} \frac{\tilde{d}}{2} \cos \left(2 \gamma_{r} d\right)}
$$


The 3-D Green's function for a 1-D periodic grid of point sources can then be written as

$$
\begin{aligned}
G_{A, V}^{\text {per }, 1-\mathrm{D}}\left(x, y, z ; x^{\prime}, y^{\prime}, z^{\prime}\right) \\
=\frac{1}{4 j} \sum_{n=1}^{+\infty} A_{n}\left(\beta_{n}, z \mid z^{\prime}\right) \sum_{m=-\infty}^{+\infty} e^{-j k_{x} m b} \\
\quad \times H_{0}^{(2)}\left(\beta_{n} \sqrt{\left(x-x^{\prime}-m b\right)^{2}+\left(y-y^{\prime}\right)^{2}}\right) .
\end{aligned}
$$

Application of the Poisson transform leads to the following equivalent series expansion

$$
\begin{aligned}
& G_{A, V}^{\mathrm{per}, 1-\mathrm{D}}\left(x, y, z ; x^{\prime}, y^{\prime}, z^{\prime}\right) \\
& =\frac{1}{2 j b} \sum_{n=1}^{+\infty} A_{n}\left(\beta_{n}, z \mid z^{\prime}\right) \\
& \quad \times \sum_{m=-\infty}^{+\infty} \frac{1}{\beta_{n, m}} e^{-j\left(\frac{2 m \pi}{b}+k_{x}\right)\left(x-x^{\prime}\right)} e^{-j \beta_{n, m}\left|y-y^{\prime}\right|}
\end{aligned}
$$

with $\beta_{n, m}=\sqrt{\beta_{n}^{2}-\left((2 m \pi / b)+k_{x}\right)^{2}}$.

\section{Convergence Analysis and Acceleration}

By applying the PML formalism, we have replaced series (2), which is a series over a single index $m$ of a spatial Green's function terms that require time-consuming Sommerfeld integration, by two equivalent series (11) and (12), over double indices $m$ and $n$, but for which the terms are easy to evaluate. Let us first concentrate on the index $n$, which runs over the different modes in the waveguide formed by the substrate together with the PMLs. In [33], the convergence of a 2-D Green's function expansion for a line source on a microstrip substrate is analyzed and it is shown that exponential convergence is obtained provided the distance $\left|y-y^{\prime}\right|$ is not too small. In a similar way, series (12) converges at a rate proportional to $\sim e^{-n C_{1}\left|y-y^{\prime}\right|}$ for $n$ large, $C_{1}$ being constant for $m$ fixed, thus exponentially as a function of $n$, yielding a rapidly converging series provided the distance $\left|y-y^{\prime}\right|$ is not too small. Series (11) converges at a rate proportional to $\sim e^{-n C_{2} \sqrt{\left(x-x^{\prime}-m b\right)^{2}+\left(y-y^{\prime}\right)^{2}}}$, for $n$ large, $C_{2}$ a constant, thus exponentially as a function of $n$, yield a fast converging series provided that either the distance $\left|x-x^{\prime}\right|$ or the distance $\left|y-y^{\prime}\right|$ is not too small. As a function of $m$, on the other hand, series (12) converges at a rate proportional to $\sim e^{-m(2 \pi / b)\left|y-y^{\prime}\right|}$, for $m$ large and for arbitrary, but fixed $n$, thus exponentially as a function of $m$, resulting in a rapidly converging series provided the distance $\left|y-y^{\prime}\right|$ is not too small. Yet, in order to obtain exponential convergence for series (11) as a function of $m$, it is required that the index $n$ is sufficiently large.
For small mode orders $n$, series (11) is slowly convergent as a function of $m$.

In [33], the Shanks transform is proposed to accelerate convergence of the PML-based mode expansion of the 2-D Green's function for a line source on a microstrip substrate. In a similar way, we apply the Shanks procedure for both series (11) and (12) in order to accelerate convergence as a function of the PML-based mode index $n$. Moreover, in this paper, for each index $n$, the Shanks transform is applied to accelerate convergence as a function of the periodicity index $m$ for both series (11) and (12). For a successful acceleration of convergence by means of the Shanks algorithm, the series must asymptotically behave as a geometric series. In general, this is not the case for (11) as a function of $m$, when considering small values of the PML-based mode order $n$ and, hence, the Shanks algorithm is not very effective in accelerating the slow convergence. Therefore, an Ewald transform is applied for mode orders $n<N$. To this end, we rewrite the periodic Green's functions as a sum of the modified spectral-domain series (12)

$$
\begin{aligned}
& G_{A, V, 1}^{\mathrm{per}, \mathrm{D}}\left(x, y, z ; x^{\prime}, y^{\prime}, z^{\prime}\right) \\
& =\frac{1}{2 j b} \sum_{n=1}^{N} A_{n}\left(\beta_{n}, z \mid z^{\prime}\right) \sum_{m=-\infty}^{+\infty} \frac{1}{\beta_{n, m}} e^{-j\left(\frac{2 m \pi}{b}+k_{x}\right)\left(x-x^{\prime}\right)} \\
& \quad \times\left[e^{+j \beta_{n, m}\left|y-y^{\prime}\right|} \operatorname{erfc}\left(\frac{j \beta_{n, m}}{2 \mathcal{E}}+\left|y-y^{\prime}\right| \mathcal{E}\right)\right. \\
& \left.\quad+e^{-j \beta_{n, m}\left|y-y^{\prime}\right|} \operatorname{erfc}\left(\frac{j \beta_{n, m}}{2 \mathcal{E}}-\left|y-y^{\prime}\right| \mathcal{E}\right)\right]
\end{aligned}
$$

and the modified spatial-domain series (11)

$$
\begin{aligned}
G_{A, V, 2}^{\mathrm{per}, 1-\mathrm{D}}\left(x, y, z ; x^{\prime}, y^{\prime}, z^{\prime}\right) \\
=\frac{1}{4 j} \sum_{q=0}^{+\infty} \frac{\sum_{n=1}^{N} \beta_{n}^{2 q} A_{n}\left(\beta_{n}, z \mid z^{\prime}\right)}{q ! 4^{q} \mathcal{E}^{2 q}} \sum_{m=-\infty}^{+\infty} e^{-j k_{x} m b} \\
\quad \times E_{q+1}\left[\mathcal{E}^{2}\left(\left(x-x^{\prime}-m b\right)^{2}+\left(y-y^{\prime}\right)^{2}\right)\right]
\end{aligned}
$$

in which $E_{q+1}[z]$ is the $q$ th-order exponential integral defined as

$$
E_{q+1}[z]=\int_{1}^{+\infty} \frac{e^{-z t}}{t^{q}} d t
$$

A suitable choice for the Ewald splitting parameter $\mathcal{E}$ has to be made. Based on the theory developed in [37] for the periodic 2-D Green's function series in free space, a suitable choice is

$$
\mathcal{E}=\max \left\{\frac{\sqrt{\pi}}{b}, \frac{\max \left[\beta_{n, 0}\right]_{n=1}^{N}}{2 H}, \frac{\max \left[\beta_{n}\right]_{n=1}^{N}}{2(\epsilon Q !)^{\frac{1}{2 Q}}}\right\}
$$

$$
A_{n}\left(\beta_{n}, z \mid z^{\prime}\right)=-\frac{2 \gamma_{r}^{2}}{\beta^{2} \epsilon_{r}^{2}} \frac{\sin \left(\gamma_{r} z\right) \sin \left(\gamma_{r} z^{\prime}\right)}{\frac{d}{\epsilon_{r}}+\tilde{d} \frac{Y_{0}^{2}+Y_{r}^{2}}{2 Y_{0}^{2}}-\frac{Y_{0} \gamma_{r} \epsilon_{r}-Y_{r} \gamma_{0}}{\gamma_{0} Y_{r}} \frac{\sin \left(2 \gamma_{r} d\right)}{2 \gamma_{r}}-\frac{Y_{0}^{2}-Y_{r}^{2}}{Y_{0}^{2}} \frac{\tilde{d} \cos \left(2 \gamma_{r} d\right)}{2}}
$$


where $H^{2}$ is the maximum exponent permitted in the spectral series (13), $\epsilon$ is the desired error, and $Q$ is the number of $q$ terms necessary to achieve convergence in the spatial series (14). Typical choices are $H^{2}=9, \epsilon=10^{-7}$, and $Q=13$ [37]. In practice, only the two lowest order TE leaky modes, the two lowest order TE Berenger modes, the two lowest order TM leaky modes, and the two lowest order TM Berenger modes are used in the Ewald accelerated series (13) and (14) so we choose $N=4$ for $G_{A}^{\text {per, } 1-\mathrm{D}}\left(x, y, z ; x^{\prime}, y^{\prime}, z^{\prime}\right)$ and $N=8$ for $G_{V}^{\mathrm{per}, 1-\mathrm{D}}\left(x, y, z ; x^{\prime}, y^{\prime}, z^{\prime}\right)$. The two series are to be complemented with either the remaining spectral-domain series

$$
\begin{aligned}
G_{A, V, N}^{\mathrm{per}, 1-\mathrm{D}}\left(x, y, z ; x^{\prime}, y^{\prime}, z^{\prime}\right) \\
=\frac{1}{4 j} \sum_{n=N+1}^{+\infty} A_{n}\left(\beta_{n}, z \mid z^{\prime}\right) \sum_{m=-\infty}^{+\infty} e^{-j k_{x} m b} \\
\quad \times H_{0}^{(2)}\left(\beta_{n} \sqrt{\left(x-x^{\prime}-m b\right)^{2}+\left(y-y^{\prime}\right)^{2}}\right)
\end{aligned}
$$

or the remaining spatial-domain series

$$
\begin{aligned}
G_{A, V, N}^{\mathrm{per}, 1-\mathrm{D}}\left(x, y, z ; x^{\prime}, y^{\prime}, z^{\prime}\right) \\
=\frac{1}{2 j b} \sum_{n=N+1}^{+\infty} A_{n}\left(\beta_{n}, z \mid z^{\prime}\right) \\
\quad \sum_{m=-\infty}^{+\infty} \frac{1}{\beta_{n, m}} e^{-j\left(\frac{2 m \pi}{b}+k_{x}\right)\left(x-x^{\prime}\right)} e^{-j \beta_{n, m}\left|y-y^{\prime}\right|}
\end{aligned}
$$

such that the complete series expansion accelerated by the Ewald procedure is given by

$$
G_{A, V}^{\mathrm{per}, 1-\mathrm{D}}=G_{A, V, 1}^{\mathrm{per}, 1-\mathrm{D}}+G_{A, V, 2}^{\mathrm{per}, 1-\mathrm{D}}+G_{A, V, N}^{\mathrm{per}, 1-\mathrm{D}} .
$$

All acceleration schemes presented up to now do not allow to calculate the 1-D periodic 3-D Green's function accurately and efficiently when both distances $\left|x-x^{\prime}\right|$ and $\left|y-y^{\prime}\right|$ are very small. Indeed, in [38], it is shown for the 2-D case that the PML-based series does not capture the correct singular behavior of $G_{A}$ at the interface of a nonmagnetic $\left(\mu_{r}=1\right)$ microstrip substrate. Therefore, we combine part of the PML-based series (11) with one term of the classical series (2) to capture the correct singularity. The following series is proposed in order to evaluate the 1-D periodic 3-D Green's function $G_{A}^{\text {per, } 1-D}$ for very small distances $\left|x-x^{\prime}\right|$ and $\left|y-y^{\prime}\right|$ at the interface:

$$
\begin{aligned}
& G_{A}^{\mathrm{per}, 1-\mathrm{D}}\left(x, y, z ; x^{\prime}, y^{\prime}, z^{\prime}\right) \\
&= G_{A}\left(x, y, z ; x^{\prime}, y^{\prime}, z^{\prime}\right) \\
&+\frac{1}{4 j} \sum_{n=1}^{+\infty} A_{n}\left(\beta_{n}, z \mid z^{\prime}\right) \sum_{\substack{m=-\infty \\
m \neq 0}}^{+\infty} e^{-j k_{x} m b} \\
& \times H_{0}^{(2)}\left(\beta_{n} \sqrt{\left(x-x^{\prime}-m b\right)^{2}+\left(y-y^{\prime}\right)^{2}}\right) .
\end{aligned}
$$

The evaluation of the Green's function $G_{A}\left(x, y, z ; x^{\prime}, y^{\prime}, z^{\prime}\right)$ for a single point source in the stratified medium is then performed by means of the classical Sommerfeld integration.

\section{EXAMPLES}

In order to assess the accuracy and efficiency of the different series expansions for the 1-D periodic 3-D Green's functions

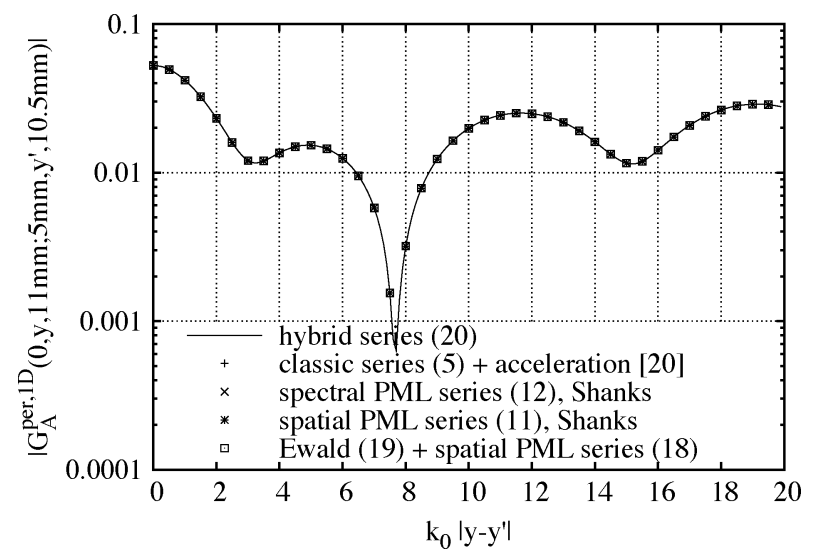

Fig. 3. Classic versus PML series for 1-D periodic 3-D Green's function $\left|G_{A}^{\text {per, } 1-\mathrm{D}}\left(0, y, 10 \mathrm{~mm} ; 5 \mathrm{~mm}, y^{\prime}, 9.5 \mathrm{~mm}\right)\right|$.

$G_{A, V}^{\text {per, } 1-\mathrm{D}}$, we consider a microstrip substrate with thickness $d=9 \mathrm{~mm}$, permittivity $\epsilon_{r}=3$, and permeability $\mu_{r}=1$. In order to obtain an expansion into PML-based modes, a closed waveguide is formed by adding a perfect electrically conducting plate above the substrate such that $d_{\text {air }}=5 \mathrm{~mm}$ and $d_{\mathrm{PML}}=$ $3.5 \mathrm{~mm}$. A strongly absorbing PML is obtained for $\kappa_{0}=15$ and $\sigma_{0} / \omega \epsilon_{0}=10$. The free-space wavelength at the operating frequency is chosen to be $\lambda_{0}=2 \mathrm{~cm}$. Based on the root-finding approach described in [35], it takes $1.56 \mathrm{~s}$ to determine the first 1000 Berenger TE modes, $2.22 \mathrm{~s}$ for the first 1000 leaky TE modes, $1.20 \mathrm{~s}$ for the first 1000 Berenger TM modes, and $1.83 \mathrm{~s}$ for the first 1000 leaky TM modes.

Let us determine the Green's functions $G_{A}^{\text {per, } 1-\mathrm{D}}\left(x, y, z ; x^{\prime}, y^{\prime}, z^{\prime}\right)$ and $G_{V}^{\text {per, } 1-\mathrm{D}}\left(x, y, z ; x^{\prime}, y^{\prime}, z^{\prime}\right)$ for a 1-D periodic set of point sources with spacing $b=1.5 \mathrm{~cm}$ (Fig. 2). The number of terms for each series evaluation is chosen in an adaptive manner in order to ensure that the relative error is smaller than $10^{-7}$. Moreover, for the PML-based series, no more than 1000 Berenger TE modes, 1000 leaky TE modes, 1000 Berenger TM modes, and 1000 leaky TM modes are taken into account. In a first numerical experiment, we investigate whether the PML sufficiently mimics the open character of the microstrip substrate by placing both excitation and observation points in the air region. The excitation point is located $0.5 \mathrm{~mm}$ above the substrate-air interface, whereas the observation point is placed at a height of $1 \mathrm{~mm}$ above the substrate-air interface. Both points are separated by a lateral distance of $\left|x-x^{\prime}\right|=5 \mathrm{~mm}$. In Fig. 3, we compare different PML-based expansions for $G_{A}^{\text {per, } 1-\mathrm{D}}\left(0, y, 10 \mathrm{~mm} ; 5 \mathrm{~mm}, y^{\prime}, 9.5 \mathrm{~mm}\right)$, choosing $k_{x}=0$ with the classic Sommerfeld integrated spectral series (2) accelerated by the method proposed in [20]. The agreement between different approaches is seen to be excellent. Specifically, for distances $\left|y-y^{\prime}\right|$ ranging from $\lambda_{0} / 600$ up to $3.2 \lambda_{0}$, the relative error between the hybrid series expansion (20) and the approach proposed in [20] remains below $0.14 \%$. Concerning $G_{V}^{\text {per, } 1-\mathrm{D}}\left(0, y, 10 \mathrm{~mm} ; 5 \mathrm{~mm}, y^{\prime}, 9.5 \mathrm{~mm}\right)$, it was found that the relative error between the hybrid series expansion (20) and the approach proposed in [20] remains even below $0.006 \%$. Hence, it is illustrated that the PML modes yield a sufficiently accurate representation of the 


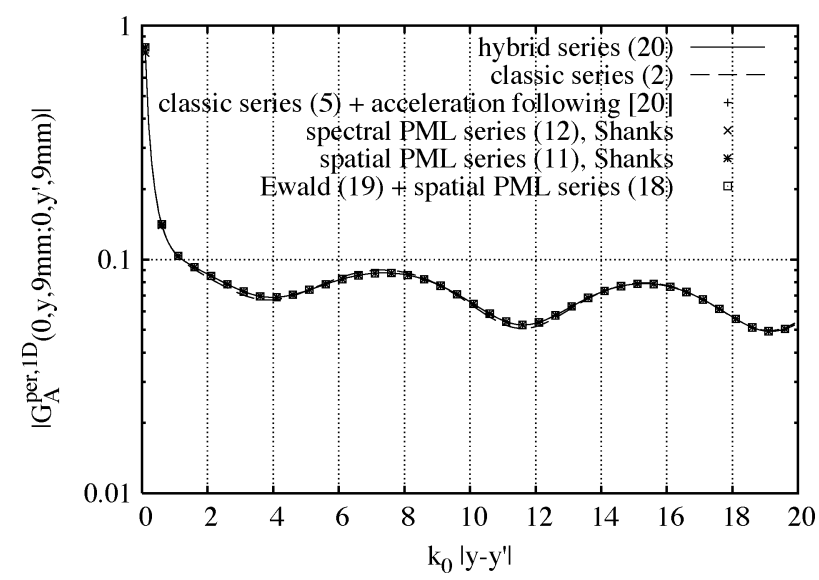

Fig. 4. Classic versus PML series for 1-D periodic 3-D Green's function $\left|G_{A}^{\text {per, } 1-\mathrm{D}}\left(0, y, 9 \mathrm{~mm} ; 0, y^{\prime}, 9 \mathrm{~mm}\right)\right|$.

evanescent spectrum in order to represent the open character of the structure.

Let us now turn our attention to a more practical situation and place both excitation and observation points at the substrate-air interface. In order to evaluate $G_{A}^{\text {per, } 1-\mathrm{D}}\left(0, y, 9 \mathrm{~mm} ; 0, y^{\prime}, 9 \mathrm{~mm}\right)$ with $k_{x}=0$ efficiently in the singularity region, i.e., for distances $\left|y-y^{\prime}\right|$ smaller than $\lambda_{0} / 60$, we make use of the hybrid series expansion (20). Comparing this result to the classic Sommerfeld integrated spectral series (2), accelerated by the method proposed in [20], a relative error smaller than $0.11 \%$ is found. In Fig. 4, the results of different series expansions for $\left|G_{A}^{\mathrm{per}, 1-\mathrm{D}}\left(0, y, 9 \mathrm{~mm} ; 0, y^{\prime}, 9 \mathrm{~mm}\right)\right|$ are shown as a function of $k_{0}\left|y-y^{\prime}\right|$, excluding the singularity by evaluating the series starting from distances of $\lambda_{0} / 60$. A small discrepancy between the classic Sommerfeld evaluation and PML-based series expansions is noticed due to the fact that the first series had not fully converged. For the classic Sommerfeld integration (2), 400 terms and Shanks acceleration were used. Yet, the classical 1-D periodic Green's function evaluation had not fully converged. However, the agreement between the PML-based series and classic Sommerfeld integration spectral series (5), accelerated by the method proposed in [20], is excellent. A more detailed view is presented in Fig. 5, where the relative error of the different series expansions is presented. The hybrid series expansion (20) is used as the reference solution since it is the only expansion that provides accurate results (convergence within a relative error smaller than $10^{-7}$ ) at all distances (the spectral series (5) is divergent for $x=x^{\prime}$ ) and it was found to be stable for a large range of PML parameters. It is found that the relative error between the hybrid series (20) and the Baccarelli approach [20] remains smaller than $0.14 \%$ for all values of $\left|y-y^{\prime}\right|$. As for the spectral-domain expansion (12) with Shanks acceleration for both indices $m$ and $n$, the relative error drops below $0.03 \%$ for distances $\left|y-y^{\prime}\right|$ larger than $\lambda_{0} / 60$. As for the spatial-domain expansion (11) with Shanks acceleration for both indices $m$ and $n$, the relative error drops below $1 \%$ for distances $\left|y-y^{\prime}\right|$ larger than $\lambda_{0} / 10$.

Concerning the different series expansions for $\left|G_{V}^{\mathrm{per}, 1-\mathrm{D}}\left(0, y, 9 \mathrm{~mm} ; 0, y^{\prime}, 9 \mathrm{~mm}\right)\right|, \quad$ a higher accuracy is obtained for the Green's function $G_{V}^{\text {per, } 1-\mathrm{D}}$ than for

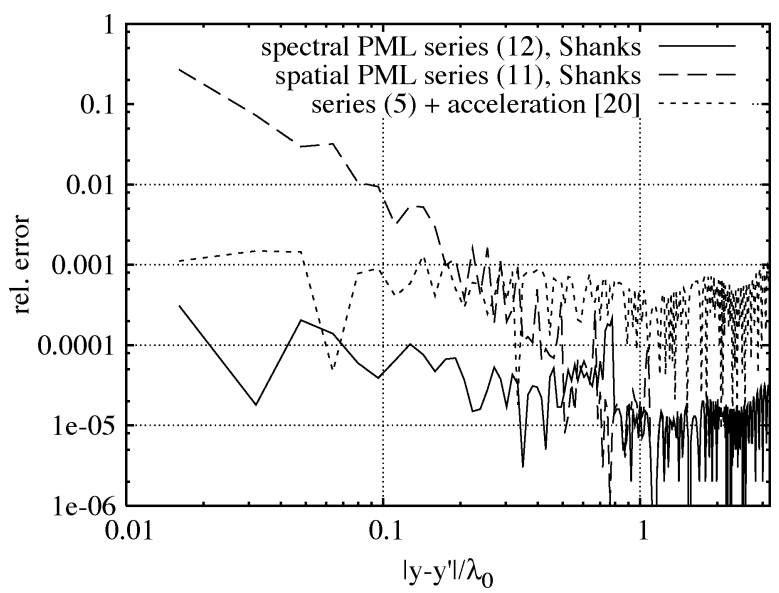

Fig. 5. Relative error of the different PML and classic expansions for $\left|G_{A}^{\text {per, } 1-\mathrm{D}}\left(0, y, 9 \mathrm{~mm} ; 0, y^{\prime}, 9 \mathrm{~mm}\right)\right|$.

TABLE I

CPU Timings FOR CAlculating 200 Points OF $\left|G_{A}^{\text {per }, 1-\mathrm{D}}\left(0, y, 9 \mathrm{~mm} ; 0, y^{\prime}, 9 \mathrm{~mm}\right)\right|$ FOR $\left|y-y^{\prime}\right| \in\left[\lambda_{0} / 60,3.2 \lambda_{0}\right]$

\begin{tabular}{|r|r|r|}
\hline Series Expansion & Acceleration technique & CPU-time \\
\hline Sommerfeld (2) & Shanks on index $m$ & $228 \mathrm{~m} 4$ s 95 \\
Baccarelli (5) & Kummer/Poisson & $2 \mathrm{~m} \mathrm{37}$ s 23 \\
spectral (12) & Shanks on index $n$ and $m$ & 0 s 89 \\
spectral (12) & Shanks on index $n$ & 0 s 46 \\
spatial (11) & Shanks on index $n$ and $m$ & $1 \mathrm{~s} 36$ \\
spatial (11) & Shanks on index $n$ & $5 \mathrm{~s} 40$ \\
hybrid (20) & Shanks on index $n$ and $m$ & $10 \mathrm{~s} 54$ \\
Ewald (19) & Series (13),(14),(18) & $1 \mathrm{~s} 17$ \\
\hline
\end{tabular}

$G_{A}^{\text {per,1-D }}$, especially near the singularity (for small values of $\left.\left|y-y^{\prime}\right|\right)$. In agreement with the theory derived in [38], all PML-based series expansions for $G_{V}^{\text {per,1-D }}$ already exhibit an accuracy better than $0.004 \%$ at distances as small as $\left|y-y^{\prime}\right|=\lambda_{0} / 60$. The discrepancy between the hybrid series (20) and the classic Sommerfeld integration spectral series (2), accelerated by the method proposed in [20], is smaller than $0.02 \%$.

In Table I, the total CPU timings are shown for calculating 200 points in the range from $\left|y-y^{\prime}\right|=\lambda_{0} / 60$ up to $\left|y-y^{\prime}\right|=3.2 \lambda_{0}$ based on the different series expansions for $\left|G_{A}^{\text {per, } 1-\mathrm{D}}\left(0, y, 9 \mathrm{~mm} ; 0, y^{\prime}, 9 \mathrm{~mm}\right)\right|$ with a relative convergence error smaller than $10^{-7}$ using a Pentium T7400 Centrino Duo 2.16-GHz machine with 2-GB RAM. It is clear that the PML-based expansions are several orders of magnitude faster than the series based on 400 conventional Sommerfeld integrations, accelerated by the Shanks algorithm. Moreover, all PML-based series evaluations are clearly much faster than the acceleration method proposed in [20] based on the classic Sommerfeld integration spectral series (2). The spectral series (12) with Shanks acceleration only performed on index $n$ is the fastest and most accurate approach. Given the exponential convergence rate proportional to $e^{-n C_{2}} \sqrt{\left(x-x^{\prime}-m b\right)^{2}+\left(y-y^{\prime}\right)^{2}}$, which leads to very fast convergence for large $m$ and for all values of $\left|x-x^{\prime}\right|$ and $\left|y-y^{\prime}\right|$, the convergence acceleration 


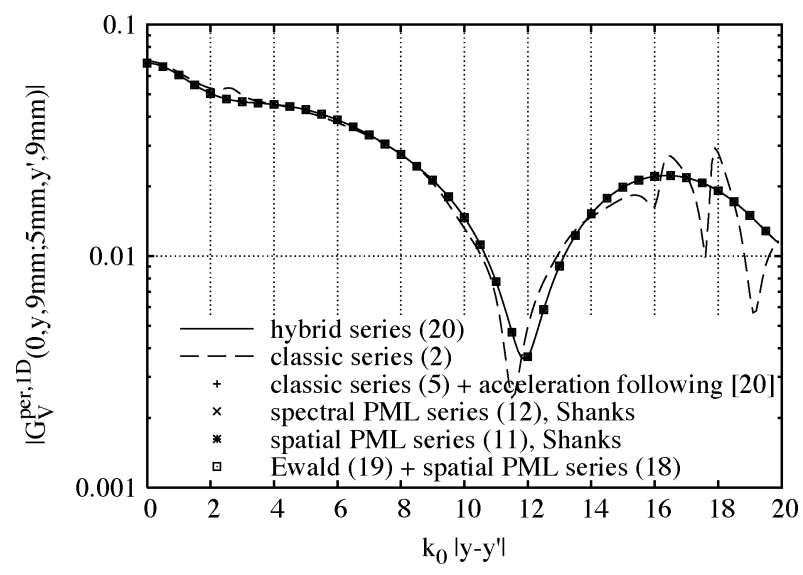

Fig. 6. Classic versus PML series for 1-D periodic 3-D Green's function $\left|G_{V}^{\text {per, } 1-\mathrm{D}}\left(0, y, 9 \mathrm{~mm} ; 5 \mathrm{~mm}, y^{\prime}, 9 \mathrm{~mm}\right)\right|$.

obtained by performing Shanks acceleration also on index $m$ is clearly insignificant and the overhead of the algorithm results in a slower evaluation procedure. For the spatial series, however, applying the Shanks transform on both indices $n$ and $m$ or using the Ewald technique by combining series (13), (14), and (18), clearly helps in terms of CPU time. In this range, however, all spatial series perform slower than the spectral PML-based series. As for the singularity region, the hybrid series expansion (20) is the preferred approach in terms of accuracy and efficiency. The evaluation of $\left|G_{A}^{\mathrm{per}, 1-\mathrm{D}}\left(0, y, 9 \mathrm{~mm} ; 0, y^{\prime}, 9 \mathrm{~mm}\right)\right|$ in the range from $\left|y-y^{\prime}\right|=\lambda_{0} / 600$ to $\left|y-y^{\prime}\right|=\lambda_{0} / 60$ based on (20) takes $0.35 \mathrm{~s}$ so the optimal evaluation based on (20) for the singularity region and (12) with Shanks acceleration performed only on index $n$ outside the singularity region takes in a total of $0.81 \mathrm{~s}$ for distances $\left|y-y^{\prime}\right|$ up to $3.2 \lambda_{0}$. Similar conclusions can be drawn for the calculation of $\left|G_{V}^{\text {per, } 1-\mathrm{D}}\left(0, y, 9 \mathrm{~mm} ; 0, y^{\prime}, 9 \mathrm{~mm}\right)\right|$ : the most optimal evaluation based on (20) for the singularity region and (12) with Shanks acceleration performed only on index $n$ outside the singularity region takes in a total $1.20 \mathrm{~s}$ for distances $\left|y-y^{\prime}\right|$ up to $3.2 \lambda_{0}$ (compared to $242 \mathrm{~m} 23 \mathrm{~s}$ for the classic series (2) with Sommerfeld integration). In Fig. 6, $\left|G_{V}^{\text {per, } 1-\mathrm{D}}\left(0, y, 9 \mathrm{~mm} ; 5 \mathrm{~mm}, y^{\prime}, 9 \mathrm{~mm}\right)\right|$ is shown as a function of $k_{0}\left|y-y^{\prime}\right|$ for a separation $\left|x-x^{\prime}\right|=5 \mathrm{~mm}$ and for $k_{x}=0.5 k_{0}$. The convergence problems with the series of 400 terms based on classic Sommerfeld integration and Shanks acceleration are again obvious, whereas an accuracy better than $0.007 \%$ is achieved for both the spectral- and spatial-domain series. The discrepancy between the hybrid series (20) and the classic Sommerfeld integration spectral series (2), accelerated by the method proposed in [20], is now smaller than $0.004 \%$.

Let us now choose a fixed distance $\left|y-y^{\prime}\right|=0$ and study the relative error for the different series expansions for $\left|G_{A}^{\text {per, } 1-\mathrm{D}}\left(x, 0,9 \mathrm{~mm} ; x^{\prime}, 0,9 \mathrm{~mm}\right)\right|$ as a function of $k_{0}\left|x-x^{\prime}\right|$, as plotted in Fig. 7 for $k_{x}=0$. Although the spectral-domain series (12) does not exhibit an exponential decay for $\left|y-y^{\prime}\right|=0$, performing the Shanks transform on both indices $m$ and $n$ yields an accuracy better than $1 \%$ as long as $\left|x-x^{\prime}\right|>\lambda_{0} / 100$. In order to obtain the same accuracy with the spatial-domain

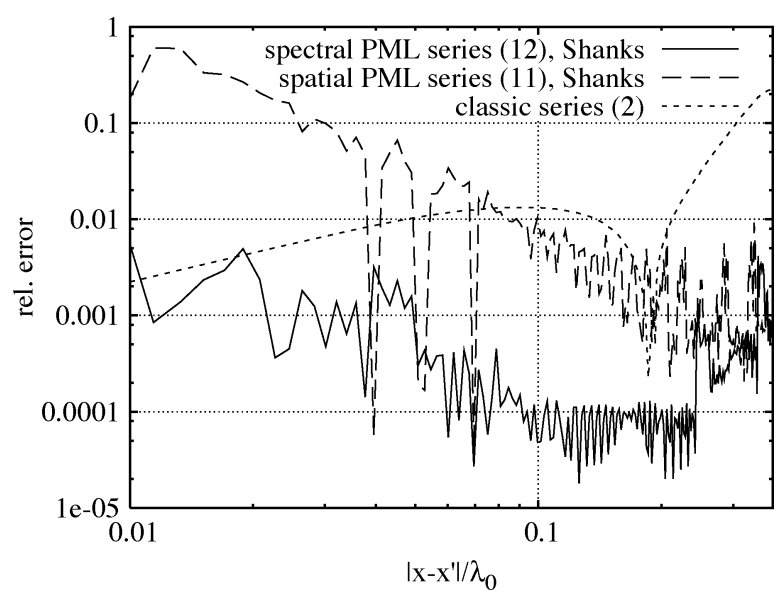

Fig. 7. Relative error of the different PML and classic expansions for $\left|G_{A}^{\text {per, } 1-\mathrm{D}}\left(x, 0,9 \mathrm{~mm} ; x^{\prime}, 0,9 \mathrm{~mm}\right)\right|$.

series (11) or with the Ewald technique by combining series (13), (14), and (18), the separation in the $x$-direction must at least be $\left|x-x^{\prime}\right|>\lambda_{0} / 10$. Yet, even with convergence acceleration by means of the Shanks algorithm, slow convergence is seen for the spectral-domain series (12) in the range from $\left|x-x^{\prime}\right|=\lambda_{0} / 100$ up to $\left|x-x^{\prime}\right|=\lambda_{0} / 10$. Within that range, it is found that the hybrid spatial series (20) is more efficient in terms of CPU time. In the range from $\left|x-x^{\prime}\right|=\lambda_{0} / 10$ to $\left|x-x^{\prime}\right|=d / 2=3 \lambda_{0} / 8$, the spectral-domain series (12) is the most efficient technique, provided that, in this case, the Shanks algorithm is applied for both indices $m$ and $n$. The optimal evaluation technique for $\left|G_{A}^{\text {per, } 1-\mathrm{D}}\left(x, 0,9 \mathrm{~mm} ; x^{\prime}, 0,9 \mathrm{~mm}\right)\right|$, based on the hybrid spatial series (20) in the singularity region and spectral-domain series (12) for $\left|x-x^{\prime}\right|>\lambda_{0} / 10$, takes $2.47 \mathrm{~s}$ of CPU time (compared to $159 \mathrm{~m} 53 \mathrm{~s}$ for the classic series (2) based on Sommerfeld integration).

As for $\left|G_{V}^{\text {per, } 1-\mathrm{D}}\left(x, 0,9 \mathrm{~mm} ; x^{\prime}, 0,9 \mathrm{~mm}\right)\right|$, the spectral-domain series (12), spatial-domain series (11), or the Ewald technique by combining series (13), (14), and (18) yield an accuracy better than $0.02 \%$ for separations in the $x$-direction of at least $\left|x-x^{\prime}\right|>\lambda_{0} / 500$. Again, slow convergence is observed for the spectral domain series (12) in the range from $\left|x-x^{\prime}\right|=$ $\lambda_{0} / 500$ up to $\left|x-x^{\prime}\right|=\left(\lambda_{0} / 10\right)$. Within that range, it is found that the hybrid spatial series (20) is more efficient in terms of CPU time. In Table II, the total CPU timings are shown for calculating 150 points based on different series expansions for $\left|G_{V}^{\text {per }, 1-\mathrm{D}}\left(x, 0,9 \mathrm{~mm} ; x^{\prime}, 0,9 \mathrm{~mm}\right)\right|$ in the range from $\left|x-x^{\prime}\right|=$ $\lambda_{0} / 10$ to $\left|x-x^{\prime}\right|=d / 2=3 \lambda_{0} / 8$ on a Pentium T7400 Centrino Duo 2.16-GHz machine with 2-GB RAM. The optimal evaluation technique for $\left|G_{V}^{\text {per,1-D }}\left(x, 0,9 \mathrm{~mm} ; x^{\prime}, 0,9 \mathrm{~mm}\right)\right|$, based on the hybrid spatial series (20) in the singularity region and spectral-domain series (12) for $\left|x-x^{\prime}\right|>\lambda_{0} / 10$, takes $3.68 \mathrm{~s}$ of CPU time.

In Fig. 8, we show the results for the Green's function series $\left|G_{A}^{\text {per, } 1-\mathrm{D}}\left(0, y, 7 \mathrm{~mm} ; 0, y^{\prime}, 7 \mathrm{~mm}\right)\right|$ inside the dielectric substrate, calculated as a function of $k_{0}\left|y-y^{\prime}\right|$ for $k_{x}=0$ and for a fixed distance $\left|x-x^{\prime}\right|=0$. The theory derived in [38] predicts that the Green's function $G_{A}$ converges to the correct singularity when evaluated inside the substrate, but not on the 
TABLE II

CPU-Timings For CAlCUlating 150 POINTS

$\left|G_{V}^{\text {per, } 1-\mathrm{D}}\left(x, 0,9 \mathrm{~mm} ; x^{\prime}, 0,9 \mathrm{~mm}\right)\right|$ FOR $\left|y-y^{\prime}\right| \in\left[\lambda_{0} / 10,3 \lambda_{0} / 8\right]$

\begin{tabular}{|r|r|r|}
\hline Series Expansion & Acceleration technique & CPU-time \\
\hline Sommerfeld (2) & Shanks on index $m$ & $145 \mathrm{~m} \mathrm{47}$ s 97 \\
spectral (12) & Shanks on index $n$ and $m$ & $2 \mathrm{~s} 15$ \\
spatial (11) & Shanks on index $n$ and $m$ & 4 s 23 \\
spatial (11) & Shanks on index $n$ & 12 s 79 \\
hybrid (20) & Shanks on index $n$ and $m$ & 6 s 3 \\
Ewald (19) & Series (13),(14),(18) & 4 s 48 \\
\hline
\end{tabular}

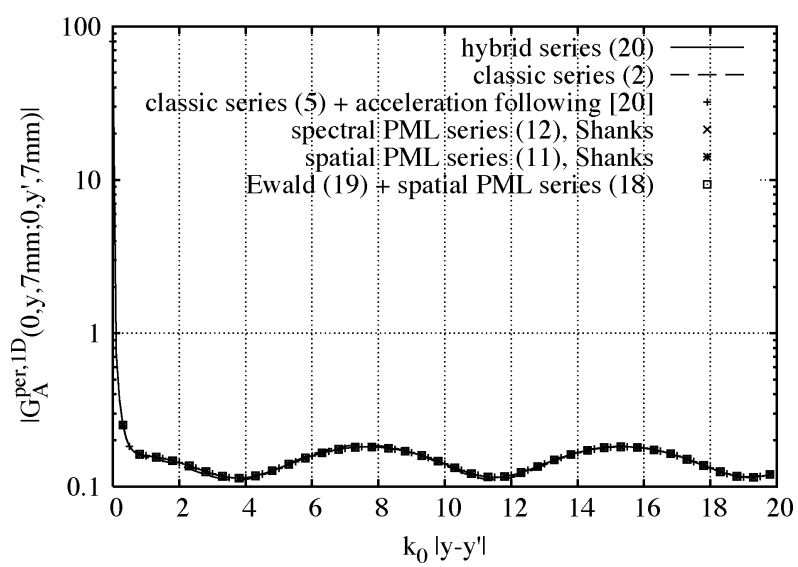

Fig. 8. Classic versus PML series for 1-D periodic 3-D Green's function $\left|G_{A}^{\text {per, } 1-\mathrm{D}}\left(0, y, 7 \mathrm{~mm} ; 0, y^{\prime}, 7 \mathrm{~mm}\right)\right|$.

interface. The resulting function near the singularity is, however, highly oscillating, thus the application of the Shanks transform is required to ensure convergence for all PML-based series expansions for $G_{A}^{\text {per,1-D }}$, resulting in relative accuracies better than $0.08 \%$ at distances as small as $\left|y-y^{\prime}\right|=\lambda_{0} / 60$. The accuracies obtained with the PML-based series expansions for $G_{V}^{\text {per, } 1-\mathrm{D}}$ are of the same order of magnitude with a relative error smaller than $0.1 \%$ at distances as small as $\left|y-y^{\prime}\right|=$ $\lambda_{0} / 60$. The optimal periodic series solution in terms of accuracy and CPU time consists of applying the hybrid series (20) in the singularity region and the spectral-domain series (12) for $\left|y-y^{\prime}\right|>\lambda_{0} / 60$. This approach takes $0.58 \mathrm{~s}$ of CPU time for $G_{A}^{\text {per, } 1-\mathrm{D}}$ and $0.96 \mathrm{~s}$ for $G_{V}^{\mathrm{per}, 1-\mathrm{D}}$. For comparison, an extension of the technique proposed in [20] takes $9 \min 9 \mathrm{~s}$ for evaluating $G_{A}^{\text {per, } 1-\mathrm{D}}$ and $18 \min 7 \mathrm{~s}$ for evaluating $G_{V}^{\mathrm{per}, 1-\mathrm{D}}$.

Finally, we study the Green's function series $\left|G_{A}^{\text {per, } 1-\mathrm{D}}\left(0, y, 9 \mathrm{~mm} ; 0, y^{\prime}, 7 \mathrm{~mm}\right)\right|$ for the excitation at the substrate-air interface and the observation point inside the dielectric substrate. In agreement with the theory derived in [38], the application of the Shanks transform is again able to ensure convergence for all PML-based series expansions for $G_{A}^{\text {per, } 1-\mathrm{D}}$. The spectral PML-based series (12) is again found to be the most accurate with a relative accuracy better than $0.2 \%$ at distances as small as $\left|y-y^{\prime}\right|=\lambda_{0} / 60$. The accuracies obtained with the PML-based series expansions for $G_{V}^{\text {per,1-D }}$ are of the same order of magnitude with a relative error smaller than $0.2 \%$ for both the spectral and spatial PML-based series at distances as small as $\left|y-y^{\prime}\right|=\lambda_{0} / 60$. The application of the hybrid series (20) in the singularity region and the spectral-domain series (12) for $\left|x-x^{\prime}\right|>\lambda_{0} / 10$ takes $0.66 \mathrm{~s}$ of CPU time for $G_{A}^{\mathrm{per}, 1-\mathrm{D}}$ and $0.88 \mathrm{~s}$ for $G_{V}^{\mathrm{per}, 1-\mathrm{D}}$. As a reference, the extension of the technique proposed in [20] takes $5 \mathrm{~min} 48 \mathrm{~s}$ for evaluating $G_{A}^{\mathrm{per}, 1-\mathrm{D}}$ and $11 \mathrm{~min} 35 \mathrm{~s}$ for evaluating $G_{V}^{\text {per, } 1-\mathrm{D}}$.

\section{Conclusions}

A new efficient and accurate formalism based on PMLs has been presented to derive new series expansions for the Green's function for both the magnetic vector potential and scalar potential of a set of point sources with 1-D periodicity embedded in a layered medium. Several PML-based series expansions, both in the spatial and spectral domain, combined with suitable convergence acceleration techniques such as the Shanks transform and Ewald transform, were examined in terms of accuracy and CPU time. In the singularity region, i.e., for the excitation and observation point locations for which

$$
\left[\frac{10\left(x-x^{\prime}\right)}{\lambda_{0}}\right]^{2}+\left[\frac{60\left(y-y^{\prime}\right)}{\lambda_{0}}\right]^{2}<1
$$

the most efficient approach is to combine the classic nonperiodic Green's function based on Sommerfeld integration with a PML-based spatial-domain series for the remaining periodic part. For all other observation/excitation pairs, the spectral-domain PML-based series with Shanks acceleration for both the $n$ and $m$ indices is the preferred approach. Future research consists of applying the new periodic series expansion to a variety of scattering and microwave applications.

\section{REFERENCES}

[1] J. Moore, H. Ling, and C. S. Liang, "The scattering and absorption characteristics of material-coated periodic gratings under oblique incidence," IEEE Trans. Antennas Propag., vol. 41, no. 9, pp. 1281-1288, Sep. 1993.

[2] P. Petre, M. Swaminathan, G. Veszely, and T. K. Sarkar, "Integral equation solution for analyzing scattering from one-dimensional periodic coated strips," IEEE Trans. Antennas Propag., vol. 41, no. 8, pp. 1069-1080, Aug. 1993.

[3] H.-C. Chu, S.-K. Jeng, and C. H. Chen, "Reflection and transmission characteristics of single-layer periodic composite structures for the TE case," IEEE Trans. Antennas Propag., vol. 45, no. 7, pp. 1065-1070, Jul. 1997.

[4] C. Craeye, A. B. Smolders, D. H. Schaubert, and A. G. Tijhuis, "An efficient computation scheme for the free space Green's function of a two-dimensional semiinfinite phased array," IEEE Trans. Antennas Propag., vol. 51, no. 4, pp. 766-771, Apr. 2003.

[5] A. Della Villa, V. Galdi, F. Capolino, V. Pierro, S. Enoch, and G. Tayeb, "A comparative study of representative categories of EBG dielectric quasi-crystals," IEEE Antennas Wireless Propag. Lett., vol. 5, pp. 331-334, 2006.

[6] G. Lovat, P. Burghignoli, F. Capolino, D. Jackson, and D. Wilton, "Analysis of directive radiation from a line source in a metamaterial slab with low permittivity," IEEE Trans. Antennas Propag., vol. 54, no. 3, pp. 1017-1030, Mar. 2006.

[7] M. G. Silveirinha and C. A. Fernandes, "Homogenization of 3-D-connected and nonconnected wire metamaterials," IEEE Trans. Microw. Theory Tech., vol. 53, no. 4, pp. 1418-1430, Apr. 2005.

[8] M. Bozzi, D. Li, S. Germani, L. Perregrini, and K. Wu, "Analysis of NRD components via the order-reduced volume-integral-equation method combined with the tracking of the matrix eigenvalues," IEEE Trans. Antennas Propag., vol. 54, no. 1, pp. 339-347, Jan. 2006. 
[9] H. Rogier, B. Baekelandt, F. Olyslager, and D. De Zutter, "The FE-BIE technique applied to some 2-D problems relevant to electromagnetic compatibility: Optimal choice of mechanisms to take into account periodicity," IEEE Trans. Electromagn. Compat., vol. 42, no. 3, pp. 246-256, Aug. 2000.

[10] E. Richalot, M. Bonilla, M. F. Wong, V. Fouad-Hanna, H. Baudrand, and J. Wiart, "Electromagnetic propagation into reinforced-concrete walls," IEEE Trans. Microw. Theory Tech., vol. 48, no. 3, pp. 357-366, Mar. 2000.

[11] D. Peña, R. Feick, H. D. Hristov, and W. Grote, "Measurement and modeling of propagation losses in brick and concrete walls for the 900-MHz band," IEEE Trans. Antennas Propag., vol. 51, no. 1, pp. 31-39, Jan. 2003.

[12] A. W. Mathis and A. F. Peterson, "A comparison of acceleration procedures for the two-dimensional Green's function," IEEE Trans. Antennas Propag., vol. 44, no. 4, pp. 567-571, Apr. 1996.

[13] S. Singh and R. Singh, "Application of transforms to accelerate the summation of periodic free-space Green's function," IEEE Trans. Microw. Theory Tech., vol. 38, no. 11, pp. 1746-1748, Nov. 1990.

[14] R. M. Shubair and Y. L. Chow, "Efficient computation of the periodic Green's function in layered dielectric media," IEEE Trans. Microw. Theory Tech., vol. 41, no. 3, pp. 498-502, Mar. 1993.

[15] R. Lampe, P. Klock, and P. Mayes, "Integral transforms useful for the accelerated summation of periodic, free-space Green's functions," IEEE Trans. Microw. Theory Tech., vol. MTT-33, no. 8, pp. 734-736, Aug. 1985.

[16] R. E. Jorgenson and R. Mittra, "Efficient calculation of the free-space periodic Green's function," IEEE Trans. Antennas Propag., vol. 38, no. 5, pp. 633-642, May 1990.

[17] H. Rogier and D. De Zutter, "A fast converging series expansion for the 2-D periodic Green's function based on perfectly matched layers," IEEE Trans. Microw. Theory Tech., vol. 52, no. 4, pp. 1199-1206, Apr. 2004.

[18] F. Capolino, D. Wilton, and W. Johnson, "Efficient computation of the 3-D Green's function with one dimensional periodicity using the Ewald method," in IEEE AP-S Int. Symp., Albuquerque, NM, Jul. 2006, pp. 2847-2850.

[19] F. Capolino, D. Wilton, and W. Johnson, "Efficient computation of the 3-D Green's function for the Helmholtz operator for a linear array of point sources using the Ewald method," J. Comput. Phys., vol. 223, pp. 250-261, Apr. 2007.

[20] P. Baccarelli, C. D. Nallo, S. Paulotto, and D. R. Jackson, "A full-wave numerical approach for modal analysis of 1-D periodic microstrip structures," IEEE Trans. Microw. Theory Tech., vol. 54, no. 4, pp. 1350-1362, Apr. 2006.

[21] F. Olyslager and H. Derruder, "Series representation of Green dyadics for layered media using PMLs," IEEE Trans. Antennas Propag., vol. 51, no. 9, pp. 2319-2326, Sep. 2003.

[22] D. Vande Ginste, E. Michielssen, D. De Zutter, and F. Olyslager, "An efficient perfectly matched layer based multilevel fast multipole algorithm for large planar microwave structures," IEEE Trans. Antennas Propag., vol. 54, no. 5, pp. 1538-1548, May 2006.

[23] P. Bienstman, H. Derudder, R. Baets, F. Olyslager, and D. De Zutter, "Analysis of cylindrical waveguide discontinuities using vectorial eigenmodes and perfectly matched layers," IEEE Trans. Microw. Theory Tech., vol. 49, no. 2, pp. 349-354, Feb. 2001.

[24] H. Derudder, F. Olyslager, D. De Zutter, and S. Van den Berghe, "Efficient mode-matching analysis of discontinuities in finite planar substrates using perfectly matched layers," IEEE Trans. Antennas Propag., vol. 49, no. 2, pp. 185-195, Feb. 2001.

[25] H. Rogier and D. De Zutter, "A fast technique based on perfectly matched layers for the full-wave space domain solution of 2-D dispersive microstrip lines," IEEE Trans. Computer-Aided Design Integr. Circuits Syst., vol. 22, no. 12, pp. 1650-1656, Dec. 2003.

[26] J. P. Bérenger, "Perfectly matched layer for the FDTD solution of wave-structure interaction problems," IEEE Trans. Antennas Propag., vol. 44, no. 1, pp. 110-117, Jan. 1996.
[27] W. C. Chew and W. H. Weedon, "A 3-D perfectly matched medium from modified Maxwell's equations in stretched coordinates," Microw. Opt. Technol. Lett., vol. 7, pp. 599-604, Sep. 1994.

[28] S. D. Gedney, "An anisotropic PML absorbing media for the FDTD simulation of fields in lossy and dispersive media," Electromagnetics, vol. 16, pp. 399-415, 1996.

[29] L. Knockaert and D. De Zutter, "On the stretching of Maxwell's equations in general orthogonal coordinate systems and the perfectly matched layer," Microw. Opt. Technol. Lett., vol. 24, pp. 31-34, Jan. 2000.

[30] L. F. Knockaert and D. De Zutter, "On the completeness of eigenmodes in a parallel plate waveguide with a perfectly matched layer termination," IEEE Trans. Antennas Propag., vol. 50, no. 11, pp. 1650-1653, Nov. 2002.

[31] F. Olyslager, "Discretization of continuous spectra based on perfectly matched layers," SIAM J. Appl. Math., vol. 64, no. 4, pp. 1408-1433, 2004.

[32] H. Derudder, F. Olyslager, and D. De Zutter, "An efficient series expansion for the 2-D Green's function of a microstrip substrate using perfectly matched layers," IEEE Microw. Guided Wave Lett., vol. 9, no. 12, pp. 505-507, Dec. 1999.

[33] H. Rogier and D. De Zutter, "Convergence behavior and acceleration of the Berenger and leaky modes series composing the 2-D Green's function for the microstrip substrate," IEEE Trans. Microw. Theory Tech., vol. 50, no. 7, pp. 1696-1704, Jul. 2002.

[34] H. Rogier and D. De Zutter, "Berenger and leaky modes in microstrip substrates terminated by a perfectly matched layer," IEEE Trans. Microw. Theory Tech., vol. 49, no. 4, pp. 712-715, Apr. 2001.

[35] H. Rogier, L. Knockaert, and D. De Zutter, "Fast calculation of the propagation constants of leaky and Berenger modes of planar and circular dielectric waveguides terminated by a perfectly matched layer," Microw. Opt. Technol. Lett., vol. 37, pp. 167-171, May 2003.

[36] L. Knockaert and H. Rogier, "An FFT-based signal identification approach for obtaining the propagation constants of the leaky modes in layered media," AEU - Int. J. Electron. Commun., vol. 59, pp. 230-238, Jun. 2005.

[37] F. Capolino, D. Wilton, and W. Johnson, "Efficient computation of the 2-D Green's function for 1-D periodic structures using the Ewald method," IEEE Trans. Antennas Propag., vol. 53, no. 9, pp. 2977-2984, Sep. 2005 .

[38] H. Rogier and D. De Zutter, "Singular behavior of the Berenger and leaky modes series composing the 2-D Green's function for the microstrip substrate," Microw. Opt. Technol. Lett., vol. 33, pp. 87-93, Apr. 2002.

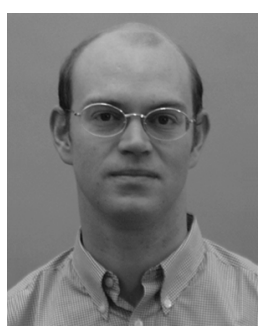

Hendrik Rogier (S'96-A'99-M'00-SM'06) was born in 1971. He received the Electrical Engineering and $\mathrm{Ph} . \mathrm{D}$. degrees from Ghent University, Gent, Belgium, in 1994 and in 1999, respectively.

He is currently a Post-Doctoral Research Fellow of the Fund for Scientific Research-Flanders (FWO-V), Department of Information Technology, Ghent University, where he is also a Part-Time Professor with the Department of Information Technology. From October 2003 to April 2004, he was a Visiting Scientist with the Mobile Communications Group, Vienna University of Technology. He has authored or coauthored approximately 35 papers in international journals and approximately 50 papers in conference proceedings. His current research interests are the analysis of EM waveguides, EM simulation techniques applied to electromagnetic compatibility (EMC), and signal integrity problems, as well as to indoor propagation and antenna design, and in smart antenna systems for wireless networks.

Dr. Rogier was a two-time recipient of the URSI Young Scientist Award presented at the 2001 URSI Symposium on Electromagnetic Theory and at the 2002 URSI General Assembly. 